\title{
Too many agencies spoil Euro research
}

Sir - Your editorial, "Dangers of Eurorelevance", urges: "In the absence of strong European alternatives, all the more reason for European governments to keep their national support for basic research" (Nature $398,1 ; 1999)$. I disagree with this conclusion which seems to want to maintain the status quo in research funding.

The European system of national research agencies hinders high-quality research. I have just reviewed, for agencies in the United Kingdom, Switzerland and Spain, grant applications that had significant overlap. As each agency uses a different pool of reviewers, such overlap is unlikely to be spotted normally, so duplicated projects are funded, wasting resources.

Even when the pressure from Republicans to decentralize the US government was at its height, the Democrats won the argument when they asked: "Do you want an Arkansas or a Montana National Institutes of Health?”. It seems that Nature advocates just such a development in European science agencies.

The pressure should instead be on the European Union to support basic science, perhaps using the dividend from the reformed Common Agricultural Policy. At a minimum, agencies should draw reviewers from all European countries, instead of only using national lists, as many still do.

\section{Carlo Gambacorti-Passerini}

Oncogenic Fusion Proteins Unit, Istituto Nazionale Tumori, Via Venezian 1, 220133 Milano, Italy

\section{Building the future of biocomputing}

Sir - It was encouraging to read the News article on the possibility that the US National Institutes of Health might invest more in computing infrastructure (Nature 398, 93-94; 1999). But, echoing Larry Smarr's comment, one wonders whether 'big iron' supercomputers are really the way to go.

Many biological calculations increasingly emphasize other aspects of computer hardware than fast central processing units for floating-point calculations. Scientists working on large-scale genome comparisons need lots of storage (on disk and in memory) and high-speed networking perhaps more than 'mega-flops'.

And those building biological databases are more concerned with the development of common data formats and easy-to-use devices for the bulk entry of laboratory data. One could even argue that these new genome- and database-oriented calculations represent a different paradigm of scientific computing from physically oriented simulations, in need of a distinct but still extensive computational infrastructure. Mark Gerstein

Molecular Biophysics \& Biochemistry Department, Yale University, Bass 432A, 266 Whitney Avenue, New Haven, Connecticut 06520-8114, USA

\section{Phenology and the}

\section{changing seasons}

Sir - Phenological data (observing seasonal plant and animal activity driven by environmental factors) have recently been used to suggest that the length of the growing season in Europe has increased over the past 30 years by perhaps a week in both spring and autumn ${ }^{1}$. These data could be interpreted somewhat differently, particularly those relating to the onset of autumn.

Clearly, the date of bud burst is a reliable indicator of the earliness of spring. To break quiescence, buds must accumulate a particular heat sum, in degree-days, above a certain threshold temperature. This is usually cited as $5^{\circ} \mathrm{C}$, presumably the point below which respiration is negligible. Climate changes occurring more than 1,000 years ago have been inferred from the recorded dates of such phenology-related events as the Kyoto cherry blossom festival ${ }^{2}$. Rarely, although perhaps increasingly frequently if the climate is getting warmer, there may be insufficient winter chilling to break rest completely, so that a late bud-burst date could mean a mild winter, not a cool spring.

Choosing a reliable phenological event with which to judge the arrival of autumn is more problematic, and dates of leaf colour change and leaf drop may be inappropriate. Various factors have been cited as causing these events, including cold, drought, photoperiod, clear weather and injury ${ }^{3}$. But leaves age ${ }^{4}$. Just as buds have a required heat accumulation for flushing, it seems that leaf life and senescence are also mediated by heat accumulation, at least in some species ${ }^{5}$. This is more likely in determinate species such as oaks, where all leaves are roughly of one age cohort ${ }^{5}$. A later end to the growing season, as judged by later leaf fall/drop dates, could be due to the fact that the leaves' heat sum is not met as early as in previous years because of later bud burst and/or cool summer temperatures, rather than a mild autumn. Incorporating summer temperatures into the model might usefully modify the conclusions about the date of the end of the growing season.

More reliable ways of assessing the effects of environment on phenology require more accurate estimates of phenological factors. The real start of spring is when mitosis starts in tissues adjacent to shoot apical meristems; the end of the growing season is when mitosis stops in the apical meristem. Unfortunately, these events are far more difficult to observe, and require destructive sampling.

\section{John Worrall}

Forest Sciences, University of British Columbia, 2424 Main Mall, Vancouver V6T 1Z4, Canada

1. Menzel, A. \& Fabian, P. Nature 397, 659 (1999).

2. Lamb, H. H. Climate Past, Present and Future (Methuen, London, 1977).

3. Kozlowski, T. T., Kramer, P. J. \& Pallardy, S. J. The Physiological Ecology of Woody Plants (Academic, New York, 1991).

4. Noodén, L. D. \& Leopold, A. C. (eds) Senescence and Aging in Plants (Academic, New York, 1988).

5. Worrall, J. J. Sust. Forestry 1, 1-18 (1993).

\section{The right prescription for preclinical teaching}

Sir - The critical and trenchant letter by the pharmacologists Hartmut Glossmann and Bernhard Peskar on the status of Austrian medical training is welcome (Nature 396, 614; 1998). But, as medical doctors, members of the teaching staff and students of the Medical School in Graz, we take issue with the statement that "Many teaching staff lack an MD qualification and do not know the basic requirements of clinical work". This statement is bewildering because, in all clinical branches, members of the teaching staff must have an MD qualification, and do know the basic requirements of clinical work.

Presumably, Glossmann and Peskar meant that in many preclinical branches biochemistry, pharmacology and physiology to name but a few - teachers of medical students do not always have an MD but instead a PhD qualification. So, the question arises whether effective and medically oriented teaching of medical students in these preclinical disciplines requires an MD qualification. In our estimation, it is not a prerequisite at all. H. Peter Soyer, Birger Kränke, Thomas Petnehazy, Stefan Scheidl Department of Dermatology,

University of Graz,

Auenbruggerplatz 8, A-8036 Graz, Austria 